\title{
The importance of an early alert from the Microbiology Laboratory and multidisciplinary collaboration during a suspected salmonellosis outbreak
}

\author{
Adriano Anesi, ${ }^{1}$ Maria Lucia Panceri, ${ }^{2}$ Sara Asticcioli, ${ }^{1}$ Dominga Baroni, ${ }^{1}$ Vanina Rognoni, ${ }^{1}$ \\ Giovanni Marazza, ${ }^{2}$ Eva Rossetti, ${ }^{2}$ Silvano Labbadini, ${ }^{3}$ Anna Archenti, ${ }^{4}$ Mario Luini, ${ }^{5}$ Barbara Bertasi, \\ Mirella Pontello, ${ }^{7}$ Annamaria Belloni ${ }^{2}$ \\ ${ }^{1}$ Microbioloy Laboratory, Maggiore Hospital, Lodi; ${ }^{2}$ Department of Medical Prevention, Local Health Unit, Lodi; \\ ${ }^{3}$ Department of Veterinary Prevention, Local Health Unit, Lodi; ${ }^{4}$ Prevention Laboratory, Local Health Unit, Milan; \\ ${ }^{5}$ IZSLER, Lodi; ${ }^{6}$ IZSLER, Brescia; ${ }^{7}$ Enterobacteria Laboratory, Department of Health Sciences, University of \\ Milan, Italy
}

\section{Summary}

Background and aims. Salmonellosis is one of the most common and widely distributed food-borne diseases. The increasing complexity and globalization of the food industry are causing an increase of some of these large-scale food-borne illnesses, thus there is a need for improvements in public health signal detection and communication streams between laboratories and regulatory agencies. The aim of this study is to show how the early reporting of salmonellosis cases directly from the Laboratory of

Correspondence: Anesi Adriano, USS Microbiologia, Ospedale Maggiore di Lodi, Largo Donatori del Sangue 1, 26900 Lodi, Italy. Tel.: +39.0371.372253 - Fax: +39.0371.372253.

E-mail: adriano.anesi@asst-lodi.it

Key words: Salmonellosis; Outbreak; Alert; Food safety; Food-borne diseases.

Contributions: AAn, MLP, design of the work, acquisition and interpretation of data for the work, drafting the work; SA, DB, VR, SL, $\mathrm{AAr}, \mathrm{ML}, \mathrm{BB}, \mathrm{MP}$, analysis and acquisition of data; GM, ER, AB, acquisition and interpretation of data for the work, revising the work critically for important intellectual content.

Conflict of interest: the authors declare no potential conflict of interest.

Received for publication: 7 September 2016.

Revision received: 9 February 2017.

Accepted for publication: 11 February 2017.

CCopyright A. Anesi et al., 2017

Licensee PAGEPress, Italy

Microbiologia Medica 2017; 32:6275

doi:10.4081/mm.2017.6275

This article is distributed under the terms of the Creative Commons Attribution Noncommercial License (by-nc 4.0) which permits any noncommercial use, distribution, and reproduction in any medium, provided the original author(s) and source are credited.
Microbiology to the Local Health Service Infectious Diseases Office along with the prompt response of the ASL, and the rapid involvement of the Local Veterinary Prevention Department resulted in an improved individuation and investigation of a suspected food-borne outbreak with anomalous manifestation.

Materials and methods. From August to November 2014 the early warning from the Laboratory of Microbiology regarding Salmonella spp. isolates with the identical serogroup and antibiotic resistance phenotype, allowed for prompt identification of a food-borne infection.

Results and conclusions. The genotyping analysis suggested that over the period considered there was more than a single monophasic Salmonella typhimurium isolate: one responsible for the sporadic cases that occurred in September and October, and another in November.

\section{Introduction}

Salmonella spp. is a bacterial agent commonly isolated in cases of infection, occasional as well as epidemic, transmitted by food (2). The increasing complexity and globalization of food production chains means that foodborne infections and food poisonings no longer show the classic epidemic peak, corresponding to a single easily identifiable episode, but can occur with outbreak patterns spreading in space and time (7). Therefore, the institutional warning systems will be decreasingly efficient, and close collaboration among various interested parties will be necessary.

The aim of this study is to demonstrate how the early reporting of salmonellosis cases directly from the Laboratory of Microbiology to the Local Health Service Infectious Diseases Office (ASL of Lodi, from 2016 merged with ATS Metropolitan City of Milan, Italy) along with the prompt response of the ASL, resulted in an improved individuation and investigation of a food-borne outbreak with anomalous manifestation. 


\section{Materials and methods}

In August 2014, the Laboratory of Microbiology of the Ospedale Maggiore of Lodi detected an anomalous increase in isolations of Salmonella spp. with the same serogroup and antibiotic resistance phenotype, and contacted the ASL. The number of early reports that arrived at the ASL from primary care physicians, provided by the Regione Lombardia infectious diseases monitoring system (1), was, however, smaller than the number of laboratory isolations.

Therefore, a close collaboration between the ASL and the Laboratory of Microbiology was agreed upon: the Laboratory would immediately and promptly report to the ASL (by phone or e-mail) the recurrence of isolations of Salmonella spp. with similar characteristics, and the ASL would undertake epidemiological investigations (based on the contact details provided by the Laboratory) for every referred case. The epidemiological investigations were conducted by phone, following a standardised form. They investigated: the symptoms in the case and in family members of the case, the foodstuffs eaten in the days preceding the first symptom, and where the foodstuffs had been purchased.

Salmonella spp. isolation from faeces samples was made following standard microbiological methods (3). Strain identifications and antibiotic susceptibility tests were performed using the Vitek2 system (BioMérieux, Marcy-l'Étoile, France); the screening of serogroups was performed with commercially available poly- and monovalent serums (Sieri test Salmonella, Siemens).

The serotyping was performed using slide agglutination tests with specific poly- and monovalent antiserums directed against somatic, flagellar and capsular antigens according to the Kauffmann-White classification (SSI Salmonella, Biogenetics).

The Salmonella typhimurium monophasic variant was determined using Multiplex PCR (5).
The qualitative research of the Salmonella spp. in foodstuffs, starting from a sample of $25 \mathrm{~g}$, was made following the ISO 6579:2002 methodology (4). The chi square test was used to assess the non-casual distribution of cases between exposed and non-exposed.

Suspecting the origin of these pathogens to be foods of animal origin, the Local Veterinary Prevention Service was promptly alerted and involved, in order to carry out controls concerning the breeding of cattle and swine, and in the main slaughterhouse of the area.

The genotype analysis of the Salmonellae isolated from faeces (made at the Enterobacteriaceae Laboratory of Milan), from foodstuff and animal samples (made at the Istituto Zooprofilattico Sperimentale Lombardia and Emilia Romagna) was conducted using pulsed field gel electrophoresis (PFGE) (6).

\section{Results}

From August to October 2014, the Laboratory of Microbiology reported to the ASL the isolation of 41 isolates of group B Salmonella spp. with the same profile of antibiotic resistance. The immediate reporting of salmonellosis from primary care physicians to the ASL occurred only in $58 \%$ of cases confirmed by the laboratory.

The epidemiological investigations of the 41 cases led to a meat product purchased in a single supermarket being suspected as the common source. The subsequent sampling of the foodstuff and in the supermarket turned out to negative.

In November 2014, the early warning from the Laboratory of Microbiology regarding a Salmonella spp. isolate, phenotypically similar (with similar antibiotic resistance profile) to the previous cluster, allowed for prompt identification of a food-borne infection of diners in an agritourism and sampling of the foodstuffs still present on-site.

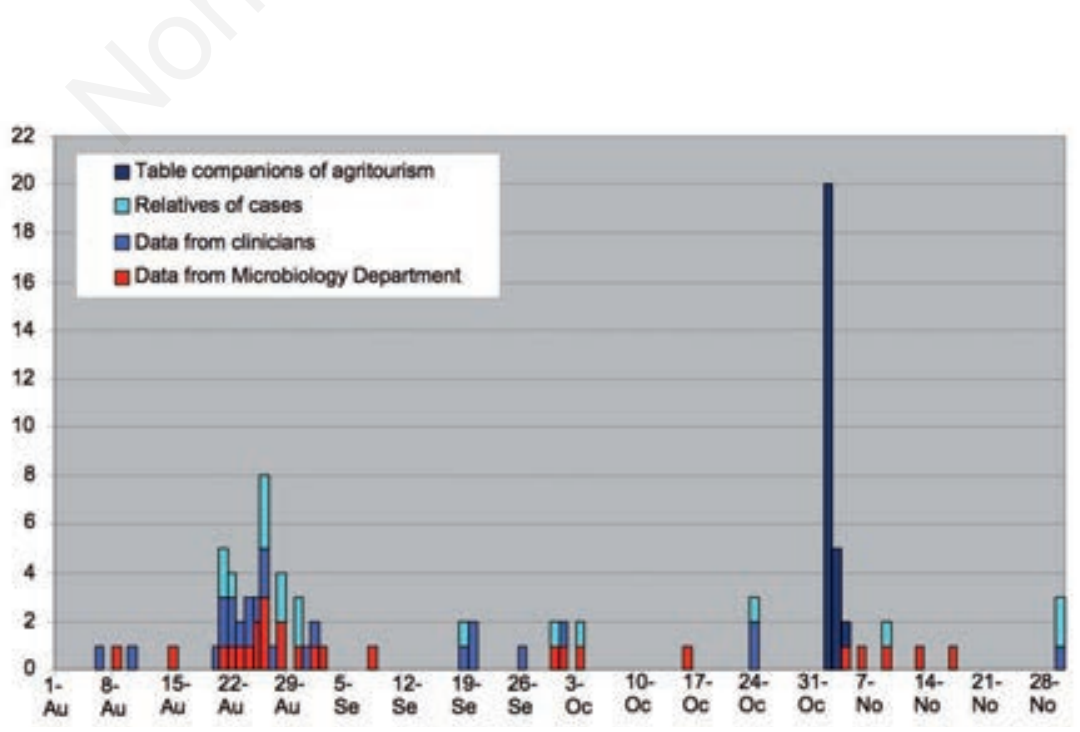

Figure 1. Cases of Salmonella spp. serogroup B with the same phenotype of antibiotic resistance from August to November 2014 according to the source of discovery. 
Figure 1 shows cases of Salmonella spp serogroup B culture confirmed from August to November 2014, according to the source of discovery, namely: infection reporting data from the Microbiology Department, notification data from clinicians, and ASL epidemiological investigations data on relatives and table companions of agritourism.

Fifty-seven diners were tested: 34 subjects showed symptoms. No foodstuff was found to be significantly associated with disease onset by the chi-square test (Table 1).

Salmonella typhimurium isolates were detected in 14 of the 18 cultures from faeces and in the food and environmental samplings from the agritourism.

The investigations revealed that the meat products involved in the two cases had been produced by two different small butchers with local customers and that the pork used by these butchers came from the same slaughterhouse, with its own system for production and distribution of meat products, approved with the CE mark, to large supermarket chains as well.

The microbiological samplings taken from the slaughterhouse highlighted the presence of monophasic Salmonella typhimurium.

The implementation of efficient cleaning and sanitisation procedures throughout the entire facility stopped the outbreak.

The genotyping of the isolates with PFGE (Table 2) was performed on seven human isolates obtained in September and on 11 isolates from food or animal source (meat product samples, bovine and swine faeces, swine carcass swabs) all obtained in November, after the food-borne infection episode took place at the agritourism.

\section{Discussion}

The analysis has pointed out that the seven human isolates belonged to the same PFGE cluster (pulsotype PR1133) and it confirms the most likely common origin of the cases. Moreover, one isolate from the meat product, one from the bovine faeces, both from the agritourism, and one of the swine isolates belonged to the same PFGE cluster pulsotype PR1133.

Other swine isolates, in particular those from meat products and suspected to be the cause of the October food-borne outbreaks, turned out to belong to a PFGE cluster pulsotype PR0004, and were thus different from the human-origin isolates found in September.

The strong similarity of these pathogens, isolated from meat products and sold by different retailers (PAN 1, 2, 3, ZAN, SAL), leads otherwise to suspect a common origin, which could be the slaughterhouse that provided the meats for the meat products in all the cases.

The genotyping analysis suggest that in the period

Table 1. Food-specific attack rates in agritourism diners.

\begin{tabular}{|c|c|c|c|c|c|c|c|c|c|c|}
\hline \multirow[t]{2}{*}{ Food item } & \multicolumn{4}{|c|}{ Food eaten } & \multicolumn{4}{|c|}{ Food not eaten } & \multicolumn{2}{|c|}{ Statistical significance } \\
\hline & Sick & Well & Total & $\begin{array}{l}\text { Attack } \\
\text { rate, } \%\end{array}$ & Sick & Well & Total & $\begin{array}{l}\text { Attack } \\
\text { rate, } \%\end{array}$ & $\begin{array}{c}\text { Attributable } \\
\text { risk }\end{array}$ & $\begin{array}{l}\text { P value } \\
\text { chi square }\end{array}$ \\
\hline Salami & 31 & 16 & 47 & 66.0 & 3 & 7 & 10 & 30.0 & 36.0 & $>0.005$ \\
\hline Cured ham & 17 & 18 & 35 & 48.6 & 17 & 5 & 22 & 77.3 & -28.7 & $>0.005$ \\
\hline Bacon & 24 & 18 & 42 & 57.1 & 10 & 5 & 15 & 66.7 & -9.5 & $>0.005$ \\
\hline Bresaola & 12 & 14 & 26 & 46.2 & 22 & 9 & 31 & 71.0 & -24.8 & $>0.005$ \\
\hline Gnocco fritto & 31 & 23 & 54 & 57.4 & 3 & 0 & 3 & 100.0 & -42.6 & $>0.005$ \\
\hline Omelette & 23 & 20 & 43 & 53.5 & 11 & 3 & 14 & 78.6 & -25.1 & $>0.005$ \\
\hline Risotto with speck & 30 & 23 & 53 & 56.6 & 4 & 0 & 4 & 100.0 & -43.4 & $>0.005$ \\
\hline Ravioli & 26 & 21 & 47 & 55.3 & 8 & 2 & 10 & 80.0 & -24.7 & $>0.005$ \\
\hline Cake ricotta and courgettes & 11 & 6 & 17 & 64.7 & 23 & 17 & 40 & 57.5 & 7.2 & $>0.005$ \\
\hline Polenta with cabbage and pork & 14 & 16 & 30 & 46.7 & 20 & 7 & 27 & 74.1 & -27.4 & $>0.005$ \\
\hline Pork fat & 24 & 20 & 44 & 54.5 & 10 & 3 & 13 & 76.9 & -22.4 & $>0.005$ \\
\hline Sausages & 16 & 15 & 31 & 51.6 & 18 & 8 & 26 & 69.2 & -17.6 & $>0.005$ \\
\hline Pork & 24 & 22 & 46 & 52.2 & 10 & 1 & 11 & 90.9 & -38.7 & $>0.005$ \\
\hline Grilled meat & 17 & 9 & 26 & 65.4 & 17 & 14 & 31 & 54.8 & 10.5 & $>0.005$ \\
\hline Salad & 8 & 13 & 21 & 38.1 & 26 & 10 & 36 & 72.2 & -34.1 & $>0.005$ \\
\hline Pie with cream & 21 & 11 & 32 & 65.6 & 13 & 12 & 25 & 52.0 & 13.6 & $>0.005$ \\
\hline Pie with ice cream & 2 & 2 & 4 & 50.0 & 32 & 21 & 53 & 60.4 & -10.4 & $>0.005$ \\
\hline Tart & 11 & 2 & 13 & 84.6 & 23 & 21 & 44 & 52.3 & 32.3 & $>0.005$ \\
\hline Ice cream & 2 & 0 & 2 & 100.0 & 32 & 23 & 55 & 58.2 & 41.8 & $>0.005$ \\
\hline Strudel & 9 & 4 & 13 & 69.2 & 25 & 19 & 44 & 56.8 & 12.4 & $>0.005$ \\
\hline Cake with pears and chocolate & 8 & 4 & 12 & 66.7 & 26 & 19 & 45 & 57.8 & 8.9 & $>0.005$ \\
\hline
\end{tabular}


Table 2. Pulsed field gel electrophoresis of Salmonella tiphymurium monophasic isolates from human, food and animal sources.

\begin{tabular}{lcccc} 
Source & Sample origin & ID & Date & Pulsotype \\
Food & Salami & PAN 1 & $17-11-2014$ & STMBX-PR0004 \\
Food & Salami & PAN 2 & $17-11-2014$ & STMBX-PR0004 \\
\hline Food & Salami & PAN 3 & $17-11-2014$ & STMBX-PR0004 \\
Food & Salami & ZAN & $19-11-2014$ & STMBX-PR0004 \\
\hline Food & Salami & SAN & $03-11-2014$ & STMBX-PR0004 \\
Food & Salami & GAL 1 & 06-11-2014 & STMBX-0132 \\
\hline Human & Feces - patient 1 & LODI 1 & September 2014 & STMBX-PR1133 \\
Human & Feces - patient 2 & LODI 2 & September 2014 & STMBX-PR1133 \\
\hline Human & Feces - patient 3 3 September 2014 & STMBX-PR1133 \\
Animal & Bovis feces & LODI 3 & $13-11-2014$ & STMBX-PR1133 \\
\hline Food & Salami & GAL 1 & $18-11-2014$ & STMBX-PR1133 \\
Animal & Swine feces & GAL 2 & S-11-2014 & STMBX-PR1133 \\
\hline Human & Feces - patient 4 & CAS & September 2014 & STMBX-PR1133 \\
Human & Feces - patient 5 & LODI 4 & September 2014 & STMBX-PR1133 \\
\hline Human & Feces - patient 6 & LODI 5 & September 2014 & STMBX-PR1133 \\
Human & Feces - patient 7 & LODI 6 & 01-12-2014 & STMBX-PR1133 \\
\hline Animal & Swine carcass & LODI 7 & 01-12-2014 & STMBX-0131 \\
Animal & Swine feces & ZAN & ZAN & STMBX-0131 \\
\hline
\end{tabular}

September-November there was more than one single monophasic Salmonella typhimurium isolate, one responsible for the sporadic cases that took place in September and October, and another, most likely different isolate, responsible for the food-borne outbreak at the agritourism.

The results obtained could not confirm a connection in the causes between the analysed meat products and the human cases, but they assume that the pork foodstuffs, especially sausages from handmade production, in which microbial starters are not used, can be contaminated by Salmonellae during slaughtering, due to the frequency with which the pigs are healthy carriers of these pathogens and many other enteric microorganisms of zoonotic relevance.

The aim of this work is not to confirm this hypothesis: not enough evidence was collected. We won't just describe a ground-breaking, innovative approach to investigating suspected food-borne outbreaks.

Using multidisciplinary and coordinated actions, such as those described here, with early involvement of the Microbiology, Public Health and Local Veterinary Prevention Departments, we can trace the origin of such complex food-borne episodes and quickly implement mechanisms of prevention.

\section{Conclusions}

Rapid and direct alerting regarding a possible salmonellosis cluster, from the Laboratory of Microbiology to the ASL, allows an epidemiological investigation to be performed more rapidly, compared to the system of infec- tious disease surveillance. The shortening of the time span between the beginning of the symptoms and the interview, makes it easier for the patients to reconstruct from memory the food history, it improves the reliability of the collected data and increases the chances of finding the suspected foodstuffs in order to perform microbiological investigations.

This work illustrates the importance of food safety and emphasizes the critical role of public health officials, clinicians, microbiologists and veterinarians in identifying and limiting (stemming) the spread of new kinds of outbreaks of food-borne disease, which are becoming ever more complicated.

\section{References}

1.D.G.R. 30 settembre 2004 N. 7/18853. Sorveglianza, notifica, controllo delle malattie infettive: revisione e riordino degli interventi di prevenzione in Regione Lombardia.

2. Hald T. Pathogen updates: Salmonella. In: Morris JG Jr., Potter ME, eds. Foodborne infections and intoxications. 4th ed. London: Academic Press; 2013. pp 67-97.

3. Isemberg HD (ed). Processing and interpretation of bacterial fecal cultures. In: Essential procedures for clinical microbiology. Washington, DC: ASM Press; 1998. pp 90-94.

4. ISO 6579:2002. Microbiology of food and animal feeding stuffs - Horizontal method for the detection of Salmonella spp.

5. Lim Y, Hirose K, Izumiya H, et al. Multiplex polymerase 
chain reaction assay for selective detection of Salmonella enterica serovar typhimurium. Jpn J Infect Dis 2003;56:151-5.

6. Ribot EM, Fair MA, Gautom R. Standardization of pulsed field gel electrophoresis protocols for the subtyping of
Escherichia coli O157:H7, Salmonella and Shigella for PulseNet. Foodborne Pathog Dis 2006;3:59-67.

7. Uyttendaele M, Franz E, Schlüter O. Food safety, a global challenge. Int J Environ Res Public Health 2016; 13:67. 